\title{
THE INVISIBILITY OF TRANSPARENT OBJECTS.
}

\author{
BY R. W. Wood.
}

\begin{abstract}
A TRANSPARENT body, no matter what its shape, disappears A when immersed in a medium of the same refractive index and dispersion. Could a transparent solid substance be found, whose refractive index and dispersion were the same as those of air, it would be absolutely invisible. We can find a solid, the refractive index of which is equal to that of air for light of a certain wave-length, but unfortunately it is not a transparent substance: If it were we should have an example of a solid absolutely invisible when illuminated with monochromatic light. The dispersion curves of some of the aniline dyes cross the line of unit refractive index for certain wave-lengths, but the point of crossing is in the immediate vicinity of the absorption band. Cases of this nature will be discussed more fully under another heading.

The disappearance of a transparent substance when immersed in a medium of identical optical properties is usually illustrated by dipping a glass rod into Canada balsam, but the disappearance is not complete, for the dispersion of the glass and the liquid are not the same. A better fluid is a solution of chloral hydrate in glycerine which is quite colorless. The glycerine is to be heated and the crystals added until the refractive index is of the required value. The hot liquid will dissolve a most astonishing amount of the chloral, ten or fifteen times its bulk, if I remember right, and only a very small amount should be placed in the beaker to start with. This solution has almost exactly the same dispersion as glass, and finely powdered glass stirred up in it becomes perfectly transparent without showing a trace of Christiansen's colors. A glass rod disappears completely when dipped into it and when withdrawn presents a curious aspect, for the end appears to melt and run freely in drops.

Lord Rayleigh in his article on optics in the Encyclopadia Brittanica points out that perfectly transparent objects are only visible
\end{abstract}


in virtue of non-uniform illumination, and that in uniform illumination they would become absolutely invisible. A condition approaching uniform illumination might, he says, be attained on a top of a monument in a dense fog. I have recently devised a method by which uniform illumination can be very easily obtained and the disappearance of transparent objects when illuminated by it illustrated. The method in brief is to place the object within a hollow globe, the interior surface of which is painted with Balmain's luminous paint and view the interior through a small hole.

The apparatus can be made in a few minutes in the following manner: A quantity of Canada balsam is boiled down, until a drop placed on cold glass solidifies. The Balmain paint, in the form of a dry powder is stirred into the hot balsam until the whole has the consistency of thick paint. Two glass evaporating dishes of equal size are carefully cleaned and warmed and coated on the outside with the hot mixture, which can be flowed over the glass, and by the dexterous manipulation of a small Bunsen flame made to cover the entire outer surface. I first tried painting the inside of a copper sphere with the commercial paint, but the surface was unsatisfactory and could not be easily cleaned. Probably two perfectly plain hemispherical finger-bowls could be used instead of the evaporating dishes. As soon as the coating has become hard a small hole is cut through it to enable the interior to be viewed. If the lips of the dishes are placed together the interior can be seen through the small opening, but in this case the line of junction, which is always more or less dark, comes opposite the aperture, which is a disadvantageous arrangement.

If the inner surfaces be exposed to bright daylight, sun or electric light, and the apparatus taken into a dark room, a crystal ball or the cut glass stopper of a decanter placed inside, it will be found to be quite invisible when viewed through the small aperture. A uniform blue glow fills the interior of the ball and only the most careful scrutiny reveals the presence of a solid object within it. One or two of the side facets of the stopper may appear if they happen to reflect or show by refraction any portion of the line of junction of the two hemispheres. 